Christian Nestler und Jan Rohgalf:

\title{
Eine deutsche Angst - Erfolgreiche Parteien rechts von der Union
}

\author{
Zur AfD und den gegenwärtigen Gelegenheitsstrukturen des \\ Parteienwettbewerbs
}

\section{Einleitung}

Das Erscheinen der »Alternative für Deutschland « (AfD) auf der politischen Bühne ist wenig überraschend und zugleich doch bemerkenswert. Angesichts der anhaltenden Krise des Euro-Raumes verwundert es nicht, dass sich - ähnlich wie in beinahe allen europäischen Ländern - auch in Deutschland eine Partei mit dezidiertem Euro-Skeptizismus ${ }^{1}$ positioniert. Nicht zuletzt stellt diese Schwerpunktsetzung ein Alleinstellungsmerkmal in der bundesdeutschen Parteienlandschaft dar. Trotzdem hat die AfD mit dem äußerst knapp verpassten Einzug in den Bundestag nicht einmal ein halbes Jahr nach der Parteigründung einen Coup gelandet. Bringt die AfD am Ende die zum politikwissenschaftlichen common sense gewordene These ins Wanken, Deutschlands politische Kultur stehe der Etablierung rechtspopulistischer Parteien entgegen?

Der Beitrag geht der Frage nach, inwiefern der Erfolg der AfD darauf zurückzuführen ist, dass der "anti-populistische Konsens ${ }^{2}$ in der alten Bundesrepublik, der auch die Wiedervereinigung weitgehend unbeschadet überstanden hat, in Auflösung begriffen ist. Dazu werden auf zwei Ebenen die Gelegenheitsstrukturen analysiert, die rechtspopulistische Parteien gegenwärtig vorfinden. Basis hierfür sind vorliegende empirische Studien zu dem Phänomen neuer Parteien, Daten der Meinungsforschung sowie eigene Auswertungen von offiziellen Parteidokumenten und Aussagen der zentralen Sprecherfiguren.

* Die Autoren danken Martin Koschkar, Jan Müller, Konstantin Sachariew und Sarah Schütt für ihre wertvollen Hinweise.

1 Die Begrifflichkeit fußt, abweichend von der angloamerikanischen Verwendung des Begriffes, auf der Trias EU-, Euro- und Europa-Skeptizismus, welche in dieser Reihenfolge aufbauend ist und eine Eskalation in der Ablehnung des Europäischen beinhaltet. Hierzu im Detail: Christian Nestler / Sarah Schütt, »Die Europawahl 2014 in Mecklenburg-Vorpommern in: Christian Nestler / Christopher Scheele (Hg.), Die Kommunalwablen 2014 in Mecklenburg-Vorpommern, Rostock 2014, S. 17-32, hier: S. 18-19.

2 Nikolaus Werz, »Erscheinungsformen und Debatten um den >Populismus < in Deutschland « in: Alexander Gallus / Thomas Schubert / Tom Thieme (Hg.), Deutsche Kontroversen. Festschrift für Eckhard Jesse, Baden-Baden 2013, S. 421-433, hier: S. 421. 
Die Ausführungen konzentrieren sich zum einen auf die Gelegenheitsstrukturen des politischen Systems. Unter dem Stichwort »neue Parteien in alten Parteiensystemen « gibt es hierzu aktuelle Untersuchungen ${ }^{3}$ die eindeutig zeigen, dass in Deutschland nur organisatorisch ausgeprägte Parteien überhaupt die Chance haben, Erfolge auf der nationalen Ebene zu konservieren. Das Wahlsystem, insbesondere die Fünf-Prozent-Hürde, sowie die Integrationskraft der etablierten Parteien erschweren das Aufkommen von »Neuen«. Dass die AfD trotzdem auf ein Mindestmaß an Erfolg blicken kann, welcher hinsichtlich des Wegfalls der Sperrklausel zur Europawahl noch angestiegen ist und mit dem Einzug in die Landesparlamente von Brandenburg, Sachsen und Thüringen einen Höhepunkt erreicht hat, ${ }^{4}$ liegt zum Teil an der sinkenden Integrationskraft der CDU/CSU rechts der Mitte und den zunehmend komplexer werdenden Politikinhalten. Ersteres resultiert aus der Anwendung des »Tina «-Prinzips unter Angela Merkel speziell im Umgang mit der Finanz- und Eurokrise ${ }^{5}$ und der schrittweisen Veränderung des Profils der Unionsparteien. Der zweite Aspekt geht auf ein Potpourri langfristiger Entwicklungen zurück, wie: Globalisierung, Individualisierung, Digitalisierung, steigende Anzahl von Akteuren in den relevanten Arenen und eine veränderte Medienöffentlichkeit. ${ }^{6}$ Dies hat ganz praktische Implikationen: Die Vermittlung der Funktionsweise des Europäischen Stabilitätsmechanismus (ESM) o. ä. passt nicht auf ein Wahlplakat, die vermeintliche Lösung für die Krise »raus aus dem Euro « hingegen schon. Damit scheint die politische Kommunikation und die Ansprache größerer, durch die Meinungsforschung auch als Euro-skeptisch erkannter ${ }^{7}$ Wählergruppen für die $\mathrm{AfD}$ relativ einfacher zu sein, als dies für andere rechtspopulistische Parteien zuvor war.

3 Nicole Bolleyer, New Parties in Old Party Systems. Persistence and Decline in Seventeen Democracies, Oxford 2013; Nicole Bolleyer / Evelyn Bytzek, Origin of party formation and new party success in advanced democracies in: European Journal of Political Research 52, Nr. 6 (2013), S. 773-796.

4 In den Statistiken des Bundeswahlleiters fällt auf, dass sich die Partei nach absoluten Zahlen lediglich von 2.056.985 auf 2.070.014 Wählern steigern konnte. In Verbindung mit der dem Abfall der Wahlbeteiligung von 71,5 Prozent bei der Bundestagswahl zu den 48,1 Prozent bei der Europawahl, hat sich das Ergebnis um 2,4 Prozentpunkte auf 7,1 Prozent erhöht.

5 Diese wird an sich bereits als »Gelegenheit « für populistische Parteien begriffen. Vgl. Florian Hartleb, Internationaler Populismus als Konzept. Zwischen Kommunikationsstil und fester Ideologie, Baden-Baden 2014, S. 159-176; Cas Mudde, »Rechtsaußen, die Große Rezession und die Europawahlen 2014 in: Aus Politik und Zeitgeschichte 64, Nr. 12 (2014), S. 16-24.

6 Siehe hierzu beispielhaft: Michael Zürn, Regieren jenseits des Nationalstaats. Globalisierung und Denationalisierung als Chance, Frankfurt/Main 1998; David Held et al., Global Transformations. Politics, Economics, Culture, Oxford 1999; Ulrich Beck, Risikogesellschaft. Auf dem Weg in eine andere Moderne, Frankfurt/Main 1986; Zygmunt Bauman, Flüchtige Moderne, Frankfurt/Main 2003; Arthur Benz (Hg.), Governance - Regieren in komplexen Regelsystemen, Wiesbaden 2004; Manuel Castells, The Information Age, 3 Bde., Chichester 2010.

7 Vgl. Renate Köcher, »Allensbach-Analyse. Alternative für Deutschland?« in: Frankfurter Allgemeine Zeitung, 17.4.2013. 
Zum anderen werden unter Rückgriff auf ein Konzept aus der sozialen Bewegungsforschung auch die »diskursiven Gelegenheitsstrukturen ${ }^{8}$ beleuchtet, aus denen die AfD Profit schlagen konnte und kann. Die Partei zehrt von, bedient und bestätigt eine Reihe von in der Gesellschaft virulenten Diskursen, die zwar nur bedingt miteinander kompatibel sind, aber alle zumindest tendenziell den anti-populistischen Konsens aufweichen: v. a. der Neoliberalismus, die Postdemokratie-Kritik und die damit einhergehenden Forderungen nach mehr direkter Demokratie, der Nationalchauvinismus, die Political Correctness-Kritik, aber auch ein neuer, gegen die »politische Klasse« gerichteter Elite-Diskurs. Als rhetorische Klammer wirkt eine ostentative Selbstviktimisierung, auf die letztlich jedweder Populismus rekurriert. Wie mit Pierre Rosanvallon argumentiert werden kann, ${ }^{9}$ ist die Selbstbezeichnung als Opfer der Mächtigen respektive der öffentlichen Meinung eine erfolgversprechende Strategie nicht nur für Rechtspopulisten, sondern allgemein in den gegenwärtigen »Gesellschaften der Partikularität«. Rechtspopulisten profitieren im Endeffekt davon, dass vor allem emanzipatorische Bewegungen im späten 20. Jahrhundert zu einer Sensibilisierung für marginalisierte Gruppen und Positionen beigetragen haben. Sie kapern gleichsam das anerkannte und moralisch hochangesehene Argumentationsmuster des Opfers der Mehrheit und der Mächtigen, um es in den Dienst einer »Propaganda der Ungleichheit ${ }^{10} \mathrm{zu}$ stellen.

Mit dem Aufzeigen der sich wandelnden Gelegenheitsstrukturen in der Bundesrepublik leistet der Artikel einen Beitrag zum Verständnis der Erfolge der AfD und des sich ändernden status quo im Parteienwettbewerb. Die Auflösung des anti-populistischen Konsenses ist durch Veränderungen in den Medien, der Kommunikation der etablierten Parteien und der Rezeption der Wähler mehr als der an die Wand gemalte Teufel. Die einfachen Lösungsvorschläge von populistischen Parteien im Allgemeinen und der AfD im Besonderen, sind als taktisches Mittel kurzfristig offenkundig chancensteigernd. Die Verstetigung dieses Erfolgs lebt in Deutschland jedoch von organisatorisch ausgeprägten Strukturen und nicht von »Führer «-persönlichkeiten. ${ }^{11}$ Nur hieraus resultiert mittelund langfristige politische Koalitions- und damit Gestaltungsfähigkeit.

8 Ruud Koopmans / Paul Statham, »Ethnic and Civic Conceptions of Nationhood and the Differential Success of the Extreme Right in Germany and Italy « in: Marco Giugni / Doug McAdam / Charles Tilly (Hg.), How Social Movements Matter, Minneapolis 1999, S. 225-251; Ruud Koopmans / Susan Olzak, » Discoursive Opportunities and the Evolution of Right-Wing Violence in Germany « in: American Journal of Sociology 110, Nr. 1 (2004), S. 198-230; Peter Ullrich, »Kulturvergleich, diskursive Gelegenheitsstrukturen und linke Nahostdiskurse. Entwurf einer wissenssoziologischen und diskurstheoretischen Perspektive für die Protestforschung « in: Rainer Keller / Ingar Truschkat (Hg.), Methodologie und Praxis der Wissenssoziologischen Diskursanalyse. Interdisziplinäre Perspektiven, Theorie und Praxis der Diskursforschung, Bd. 1, Wiesbaden 2012, S. 315-337.

9 Pierre Rosanvallon, Counter-democracy. Politics in an Age of Distrust, Cambridge u.a. 2008; ders., Demokratische Legitimität. Unparteilichkeit - Reflexivität - Nähe, Hamburg 2010.

10 Albrecht von Lucke, »Propaganda der Ungleichheit« in: Blätter für deutsche und internationale Politik 54, Nr. 12 (2009), S. 55-63.

11 Bolleyer, New Parties in Old Party Systems. Persistence and Decline in Seventeen Democracies, aaO. (FN 3), S. 41; Cas Mudde, Populist Radical Right Parties in Europe, Cambridge 2007. 


\section{Gelegenheitsstrukturen}

Nicht anders als Machiavellis Fürst ${ }^{12}$ sind auch die politischen Akteure in den gegenwärtigen Demokratien in ihrem Handeln mit externen Faktoren konfrontiert, die sie in nur sehr begrenztem Maße verändern können. Da diese Faktoren als Hindernisse oder günstige Gelegenheiten jedoch maßgeblich für Erfolg oder Misserfolg sein können, tun politische Akteure gut daran, sie genau zu kennen und in ihren Handlungen in Rechnung zu stellen.

Für die westlichen Demokratien wurde diese Handlungsanweisung in Form der »strukturierten Gelegenheiten « nach Robert Merton operationalisiert. ${ }^{13}$ Dieser kriminalsoziologische Ausgangspunkt war unter anderem die Basis für die Theorie der »politischen Gelegenheitsstrukturen «.

Den Ausführungen von Cas Mudde folgend, ist eine angebotsseitige Darstellung der Gelegenheiten für rechtspopulistische Parteien an die Teilung in institutionelle, politische und kulturelle Kontexte gebunden (2.1). Dabei wird auf die getrennte Darstellung von externer und interner Angebotsseite verzichtet. Der diskursive und damit der mediale Kontext wird gesondert behandelt (2.2). Die folgende Analyse teilt sich daher in »institutionelle, politische und kulturelle« (3.) sowie »diskursive« (4.) Gelegenheitsstrukturen. ${ }^{14}$

Der Teilung liegt die pragmatische Überlegung zugrunde, dass neben den vorhandenen Rahmenbedingungen des politischen Systems und dessen Wettbewerb - Wahlsystem, Parteienfinanzierung etc. - für eine neue Partei gerade der öffentliche Diskurs für den Erfolg von entscheidender Bedeutung ist. Erstes strategisches Ziel ist es zwangsläufig dem eigenen Namen (positiv) im Wahlvolk zu verankern.

\subsection{Institutionelle Gelegenheitsstrukturen}

Das Konzept der »politischen Gelegenheitsstrukturen« wurde allgemein in der Soziologie und im Besonderen in der Bewegungs- und Protestforschung entwickelt. Der so genannte Political Opportunity Structure-Ansatz gehört zu den Standards der Disziplin. Er geht, vereinfacht gesagt, davon aus, dass für die Form, das Aktionsrepertoire und das Handeln sozialer Bewegungen die wahrgenommenen Optionen und deren Erfolgsaussichten maßgeblich sind, die das politische System bereithält. Zu diesen Faktoren werden üblicherweise etwa die Responsivität des politischen Systems gezählt, die Struktur von

$12 »[\ldots]$ wenn einer sich mit Vorsicht und Geduld benimmt und die Zeitumstände derart sind, daß seine Handlungsweise gut ist, so gelingt ihm sein Vorhaben; ändern sich aber die Verhältnisse, so geht er zugrunde, weil er seine Handlungsweisen nicht ändert. «, Niccolò Machiavelli, Der Fürst, Frankfurt am Main 1990, S. 119.

13 Robert K. Merton, »Social Structure and Anomie« in: American Sociological Review 3, Nr. 5 (1938), S. 672-682; ders., »Opportunity Structure: The Emergence, Diffusion, and Differentiation of a Sociological Concept, 1930s-19950s « in: Freda Adler / William Laufer (Hg.), The Legacy of Anomie Theory, New Brundswick 1995, S. 3-78.

14 Vgl. Mudde, Populist Radical Right Parties in Europe, aaO. (FN 11), S. 232-276. 
Eliten oder die Wahrscheinlichkeit und Härte staatlicher Repressionen. ${ }^{15}$ Damit handelt es sich grundsätzlich um eine rationalistische Deutung eines »außerparlamentarischen, Regeln verletzenden politischen Aktivismus. Dieser Aktivismus kommt demnach in Gang, wenn der betroffenen Gruppe aus sstrukturellen< Gründen der Weg versperrt ist, ihre politischen Ziele auf konventionellem Weg zu verfolgen.«16

Neuen Parteien kann der Weg nicht im eigentlichen Sinne versperrt werden. Die Gründung dieser ist in Deutschland frei und nur durch im Parteiengesetz festgehaltene Formalia eingeschränkt. ${ }^{17}$ »Verhinderung « passiert in Deutschland im Regelfall durch Hürden im System, ${ }^{18}$ die Persistenz und Integrationskraft der etablierten Parteien und die politische Kultur. Gleichwohl gilt Mertons Aussage, dass: »Opportunity structure designates the scale and distribution of conditions that provide various probabilities for individuals and groups to achieve specifiable outcomes. From time to time, the opportunity structure expands or contracts. ${ }^{19}$ Mit Blick auf die Entwicklung neuer Parteien in Deutschland spiegelt sich dieser Gedanke in der als Angebotsseite bezeichneten Dimension des Parteienwettbewerbs wider. Wirkungsmächtiger und empirisch nachweisbarer Einfluss entsteht allerdings erst im Zusammenspiel mit der Nachfrageseite und den landeseigenen Kontextbedingungen. ${ }^{20}$ Für die parlamentarische Demokratie der Bundesrepublik hat Oskar Niedermayer vor dem Hintergrund des Aufstiegs der Piratenpartei zwischen 2009 und 2012 diese drei Seiten mit ihren Faktoren erschöpfend dargestellt (siehe Tabelle 1). ${ }^{21}$

15 Siehe u. a. Sidney Tarrow, Power in Social Movements. Collective Action and Politics, Cambridge 1994, v. a. S. 71-90; Doug McAdam / Sidney Tarrow / Charles Tilly, Dynamics of Contention, Cambridge 2001; Donatella della Porta / Mario Diani, Social Movements. An Introduction, Oxford 1999, S. 193-225; Ruud Koopmans, »Political Opportunity Structure. Some Splitting to Balance the Lumping « in: Sociological Forum 14, Nr. 1 (1999), S. 93-105.

16 Andreas Pettenkofer, Radikaler Protest. Zur soziologischen Theorie politischer Bewegungen, Frankfurt a.M./New York 2010, S. 45.

17 Vgl. Heike Merten, »Rechtliche Grundlagen der Parteiendemokratie« in: Frank Decker / Viola Neu (Hg.), Handbuch der deutschen Parteien, Wiesbaden 2013, S. 77-110, hier: S. 82f. Ausnahmen von dieser Regel sind hier selbstverständlich Parteienverbotsverfahren.

$18 \mathrm{Zu}$ den Hürden, die neue Parteien überwinden müssen, gibt es inzwischen einige - zum Teil aufeinander aufbauende - Modelle. Diese bilden letztlich die Basis für die frage nach den Erfolgsbedingungen für (neue) Parteien. Siehe u.a. Seymour Martin Lipset / Stein Rokkan, »Cleavage Structures, Party System, and Voter Alignments: An Introduction « in: dies. (Hg.), Party Systems and Voter Alignments. Cross-national Perspectives, New York 1967, S. 1-64; Oskar Niedermayer, »Einleitung: Erfolgsbedingungen neuer Parteien im Parteiensystem « in: ders. (Hg.), Die Piratenpartei, Wiesbaden 2013, S. 7-14; Ferdinand Müller-Rommel, Grüne Parteien in Westeuropa. Entwicklungsphasen und Erfolgsbedingungen, Opladen 1993; Mogens N. Pedersen, »Towards a New Typology of Party Lifespans and Minor Parties « in: Scandinavian Political Studies 5, Nr. 1 (1982), S. 1-16.

19 Merton, »Opportunity Structure: The Emergence, Diffusion, and Differentiation of a Sociological Concept, 1930s-1950s«, aaO. (FN 13), S. 25.

20 Vgl. Mudde, Populist Radical Right Parties in Europe, aaO. (FN 11), S. 255.

21 Vgl. Oskar Niedermayer, »Erfolgsbedingungen neuer Parteien im Parteiensystem am Beispiel der Piratenpartei Deutschland « in: Zeitschrift für Parlamentsfragen 41, Nr. 4 (2010), S. 838-854; Niedermayer, »Einleitung: Erfolgsbedingungen neuer Parteien im Parteiensystem«, aaO. (FN 18), S. $9 \mathrm{ff}$.

ZfP 61. Jg. 4/2014 
Tabelle 1: Die drei Dimensionen des Parteienwettbewerbs nach Oskar Niedermayer

\begin{tabular}{|l|l|}
\hline Dimension & \\
\hline Angebotsseite & $\begin{array}{l}\text { - Ressourcen der neuen Partei und ihrer Konkurrenz } \\
\text { - Strategie der neuen Partei und ihrer Konkurrenz }\end{array}$ \\
\hline - Politikinhalte der neuen Partei und ihrer Konkurrenz
\end{tabular}

Quelle: Eigene Darstellung nach Niedermayer. ${ }^{22}$

Für einen neuen Akteur im Parteienwettbewerb sind nun die institutionellen, politischen und kulturellen Eigenheiten, die diese Organisation vorfindet, zu spezifizieren. Diese idealtypische Darstellung zielt dabei auf die Besonderheiten, der sich eine hypothetische rechtspopulistische Partei stellen muss. ${ }^{23}$ Dabei kann in der Reflektion der vorhandenen Literatur festgestellt werden, dass (1) in Deutschland die Etablierung neuer Parteien prinzipiell selten ist ${ }^{24}$ und dass (2), anders als ideologisch mitte-links aufgestellte Parteien, rechte und rechtspopulistische Parteien im Wettbewerb durch die politische Kultur Deutschlands eine eigenständige Hürde vor sich haben. ${ }^{25}$

22 Ebd., S. 9ff.

23 Mudde, Populist radical right parties in Europe, aaO. (FN 11), S. 201-276; ders., »The Populist Radical Right: A Pathological Normalcy in: West European Politics 33, Nr.6 (2010), S. 1167-1186; ders., »The 2012 Stein Rokkan Lecture. Three decades of populist radical right parties in Western Europe in: European Jorunal of Political Research 52, Nr. 1 (2013), S. 1-19.

24 Vgl. Bolleyer, New Parties in Old Party Systems. Persistence and Decline in Seventeen Democracies, aaO. (FN 3), S. 38.

25 Frank Decker / Florian Hartleb, »Populismus auf schwierigem Terrain. Die rechten und linken Herausfordererparteien in der Bundesrepublik « in: Frank Decker (Hg.), Populismus. Gefahr für die Demokratie oder nützliches Korrektiv?, Wiesbaden 2006, S. 191-215; Frank Decker, Wenn die Populisten kommen. Beiträge zum Zustand der Demokratie und des Parteiensystems, Wiesbaden 2013. 
Der erste Punkt zeigt sich unzweideutig in der Geschichte des deutschen Parlamentarismus seit 1945. Nach der Konzentrationsphase des Parteiensystems ab 1953 gab es nur zwei erfolgreiche Neugründungen wobei Bündnis 90/Die Grünen und die Die LINKE programmatisch beide auf dem erweiterten linken Spektrum zu verorten sind. ${ }^{26}$ Die Beispiele auf der rechten Seite - DVU, NPD, Republikaner, Schill-Partei - verdeutlichen den zweiten Punkt. Diese Parteien hatten oder haben nur temporäre und lokal begrenzte Erfolge. Der Kontext des Nationalsozialismus zwischen 1933 und 1945, der anti-totalitäre Konsens der jungen Bundesrepublik, das Verbot der Sozialistischen Reichspartei 1952 sowie die zwei Verbotsanträge gegen die NPD sind in dieser Hinsicht bis heute wirkungsmächtige Einflüsse in der politischen Kultur und zum Teil selbst Ausdruck derselben. ${ }^{27}$

Resümierend ist in Bezug auf Mertons Aussage, dass sich Gelegenheitsstrukturen über die Zeit vergrößern und verkleinern, ${ }^{28}$ für die drei hier betrachteten Aspekte der Angebotsseite zu sagen, dass diese, vernachlässigt man ausgesprochen langfristige Entwicklungen und Gesetzesänderungen, relativ statisch sind. Gleichwohl zeigt die viel diskutierte Absenkung und dann Abschaffung ${ }^{29}$ der Sperrklausel zur Europawahl 2014 durch das Bundesverfassungsgericht, wie schnell eine Gelegenheit entstehen kann.

\subsection{Diskursive Gelegenheitsstrukturen}

Die diskursiven Gelegenheitsstrukturen, die nun die Akteure der AfD vorfinden und welche entscheidend für ihren Erfolg sind, können wiederum im Anschluss an die soziale Bewegungsforschung konzeptualisiert werden. Der niederländische Soziologe Ruud Koopmans hat, in Erweiterung der oben skizzierte Faktorenanalyse, vorgeschlagen, den Diskurs einzubeziehen, um insbesondere der Bedeutung von Massenmedien für politi-

26 Gleichzeitig zeigen diese neuen Akteure die Integrationskraft der Unionsparteien, welche, anders als die SPD, bisher keine "Spaltung « erleben musste. Vgl. Andreas M. Vollmer, Arbeit $\mathcal{E}$ soziale Gerechtigkeit - Die Wablalternative (WASG). Entstehung, Geschichte und Bilanz, Baden-Baden 2013, S. 45-60.

27 Siehe u. a.: Eckhard Jesse, »Antiextremistischer Konsens. Von der Weimarer Republik bis zur Gegenwart « in: Karl G. Kick / Stephan Weingarz / Ulrich Bartosch (Hg.), Wandel durch Beständigkeit. Studien zur deutschen und internationalen Politik. Jens Hacker zum 65. Geburtstag, Berlin 1998, S. 151-169; Michael Oswald, »Verbot trotz Bedeutungslosigkeit? Die NPD, ihre Strategie und die Erfolgsaussichten des neuen Verbotsantrags « in: Zeitschrift für Parlamentsfragen 45, Nr. 2 (2014), S. 440-460; Kurt Sontheimer, Antidemokratisches Denken in der Weimarer Republik. Die politischen Ideen des deutschen Nationalismus zwischen 1918 und 1933, München 1992.

28 Vgl. Merton, »Opportunity Structure: The Emergence, Diffusion, and Differentiation of a Sociological Concept, 1930s-1950s«, aaO. (FN 13), S. 25.

29 Bundesverfassungsgericht: Entscheidung 2 BvE 2/13, 26.2.2014.

ZfP 61. Jg. 4/2014 
sche Akteure in den gegenwärtigen Demokratien gerecht zu werden. ${ }^{30}$ Dabei unterscheidet er drei Elemente diskursiver Gelegenheiten. ${ }^{31}$

Sichtbarkeit. »Was wir über unsere Gesellschaft, ja über die Welt, in der wir leben, wissen, wissen wir durch die Massenmedien «. ${ }^{32}$ Dies bedeutet für politische Akteure, dass ihre Existenz ganz massiv von der Präsenz in den Massenmedien abhängt. Die öffentliche Relevanz von Akteuren, Ereignissen und Botschaften entspricht (weitgehend) der Relevanz, die ihr in den Medien beigemessen wird. Deshalb müssen sich die politischen Akteure mit ihren Botschaften in einem Konkurrenzkampf mit anderen (nicht nur politischen) Botschaften um knappe Sendezeiten, um Zeitungsspalten und insbesondere um die begrenzte Aufmerksamkeit des Publikums durchsetzen können, wobei die Chancen und Ressourcen der Wettbewerber ungleich verteilt sind.

Resonanz. Inwiefern provoziert eine Botschaft wiederum Reaktionen in den Medien? Wird eine Botschaft in den Zeitungen kommentiert? Werden ihre Urheber zu Talkshows eingeladen? Oder nehmen andere politische Akteure Bezug auf diese Botschaft? Dabei kann naheliegenderweise die Zustimmung von prominenter Seite der Relevanz einer Botschaft und ihres Urhebers zu Gute kommen. Genauso unterstreicht aber auch die öffentliche Ablehnung letztlich die Relevanz der Botschaft und erhöht so auch deren Sichtbarkeit. Gerade das Beispiel der AfD zeigt, wie die öffentliche Ablehnung und Empörung sogar in eine wichtige strategische Ressource umgewandelt werden kann.

Legitimität. Gibt es mehr Zustimmung als Gegenworte? Freilich trachten politische Akteure danach, als achtbare Akteure mit legitimen Anliegen wahrgenommen zu werden. Aber eine überaus hohe Legitimität kann auch bedeuten, dass eine Botschaft keine nennenswerte Sichtbarkeit erlangt, weil sie zu sehr als common sense gilt, als dass ihr noch ein Nachrichtenwert beigemessen wird. Gerade die Kontroversität einer Botschaft wird gewöhnlich mit erhöhter (Medien-)Aufmerksamkeit belohnt. Interessant ist bei der AfD, dass ihre Wortführer äußerst selektiv den Unterschied zwischen öffentlicher und veröffentlichter Meinung bemühen, um gerade die Kritik an der Partei in den Medien nicht ohne Erfolg - in ein Indiz für die Legitimität ihrer Anliegen umzudeuten.

Die Beziehung zwischen Gelegenheitsstrukturen und Akteuren ist weniger eindeutig, als ein flüchtiger Blick vielleicht vermuten lassen mag. Schließlich können Akteure, die eine Gelegenheit beim Schopfe packen, sich auf diesem Wege auch neue Optionen verschaffen oder vormals noch vorhandene verspielen. Kurz: Das Handeln der Akteure kann sehr wohl auf die Gelegenheitsstruktur zurückwirken. Dies trifft in noch größerem Maße auf die diskursiven Gelegenheitsstrukturen, als auf die institutionellen zu. Bisweilen kann schon ein einzelner geschickt platzierter Beitrag zu einem in den Medien bereits kontrovers verhandelten Thema ausreichen, um der Diskussion eine neue Richtung zu geben und dem Urheber des Beitrags zumindest kurzfristig die Aufmerksamkeit des Publikums zu sichern.

30 Zuerst in: Koopmans / Statham, »Ethnic and Civic Conceptions of Nationhood and the Differential Success of the Extreme Right in Germany and Italy «, aaO. (FN 8), S. 225-251.

31 Zum Folgenden: Koopmans / Olzak, »Discoursive Opportunities and the Evolution of RightWing Violence in Germany", aaO. (FN 8), S. 198-230.

32 Niklas Luhmann, Die Realität der Massenmedien, Wiesbaden 2009, S. 9. 
Während der Framing-Ansatz ${ }^{33}$ die Deutungsrahmen untersucht, in die politische Akteure ihre Botschaften einbetten, um eine bestimmte Resonanz zu erwirken, wird mit den diskursiven Gelegenheitsstrukturen gleichsam die andere Seite beleuchtet. Das Augenmerk verschiebt sich auf die Situation, in die hinein die politischen Akteure ihre Botschaften senden, auf die Deutungsrahmen, Themen und Diskurse, die erfolgreiche Framing-Akte aufgreifen. ${ }^{34}$ Dementsprechend wird in diesem Artikel argumentiert, dass Sprecherfiguren der AfD an eine Reihe von Diskursen anknüpfen, die derzeit in der Öffentlichkeit in Deutschland virulent sind. Dadurch, dass die Wortführer bestimmte Begriffe, Argumentationsmuster und sprachliche Bilder aufgreifen, wenn sie ihre politischen Botschaften verbreiten, kann es ihnen möglich sein, potentielle Unterstützer- und Wählerkreise mit unterschiedlichen Anliegen und Hintergründen gleichermaßen anzusprechen.

Ohne Frage sind strategisches Handeln und gezielte Manipulation Bestandteil der politischen Wirklichkeit. Und sicher muss nach den Zielen und Interessen gefragt werden, die Akteure verfolgen. Allerdings lohnt es sich auch, die öffentlichen Äußerungen politischer Akteure nicht allein als strategische Einlassungen zu betrachten. Es ist davon auszugehen, dass individuelle Akteure mit der Politik auch ihre eigenen Interessen - etwa Macht, Ansehen, Geld ${ }^{35}$ - verfolgen. Jedoch ist das Engagement für dieses oder jenes politische Projekt in der Regel nicht vollends von derartigen Überlegungen bestimmt, sondern auch von der Affinität zu bestimmten Inhalten. ${ }^{36}$

Um auch dem Fall gerecht zu werden, dass Akteure an das glauben, was sie sagen, empfiehlt Peter Ullrich eine alternative Lesart der diskursiven Gelegenheitsstrukturen. In Anlehnung an die wissenssoziologische Diskurstheorie Rainer Kellers ${ }^{37}$ lenkt er den Fokus darauf, dass die vorfindlichen Deutungsmuster für die politischen Akteure nur bedingt disponibel sind. Vielmehr haben die Akteure selbst bestimmte Deutungsmuster

33 Vgl. David Snow et al., »Frame Alignment Processes, Micromobilization and Movement Participation « in: American Sociological Review 51, Nr. 4 (1986), S. 464-481; David Snow / Robert D. Benford, »Ideology, frame resonance and participant mobilization « in: International Social Movement Research 1, Nr. 1 (1988), S. 197-217.

34 Koopmans / Olzak, »Discoursive Opportunities and the Evolution of Right-Wing Violence in Germany «, aaO. (FN 8), S. 199.

35 Wie etwa in Anthony Downs' realistischer Demokratie-Theorie Interessen definiert werden, Anthony Downs, "An Economic Theory of Political Action in a Democracy « in: Journal of Political Economy 65, Nr. 2 (1957), S. 135-150.

36 Zudem impliziert die Annahme der rein strategischen oder gar manipulativen politischen Rede die wenig überzeugende Konstellation, in der einer politischen Elite, die ein zynisches, rein instrumentelles Verhältnis zu den politischen Inhalten pflegt, ein Fußvolk gegenüber steht, das diese für bare Münze nimmt. Ein ähnlicher bias findet sich auch in den klassischen Texten zur symbolischen Politik wie Harold Lasswell, »Politics: Who gets what, when, how « in: ders., The Political Writings, Glencoe 1951, S. 295-461 oder Murray Edelman, Politik als Ritual. Die symbolische Funktion staatlicher Institutionen und politischen Handelns, Frankfurt am Main/ New York 1990.

37 Ullrich, »Kulturvergleich, diskursive Gelegenheitsstrukturen und linke Nahostdiskurse. Entwurf einer wissenssoziologischen und diskurstheoretischen Perspektive für die Protestforschung «, aaO. (FN 8), S. 315-337; Rainer Keller, Wissenssoziologische Diskursanalyse. Grundlegung eines Forschungsprogramms, Wiesbaden 2011.

ZfP 61. Jg. 4/2014 
verinnerlicht, die nur schwer zu verändern sind. Diese formen maßgeblich, was Akteure für wahr und wertvoll erachten, also deren Selbstverständnis. Die verinnerlichten Deutungsmuster beeinflussen als Filter gleichermaßen die Bewertung von Problemlagen und die Wahrnehmung verfügbarer Handlungsoptionen und -motive.

Vom diskursiven Habitat der AfD ist im Folgenden die Rede, weil sowohl die Vorstellungen und Diskurse interessieren, in denen die tonangebenden Politiker der Partei »zu Hause « und verwurzelt sind als auch das diskursive Umfeld, auf das sie rekurrieren. Freilich lassen sich beide Fälle nicht immer sauber auseinanderhalten. Im Rahmen dieses Aufsatzes wird lediglich auf zwei Diskurse eingegangen, in denen Sprecherfiguren der Partei seit längerem engagiert sind und die in einem Spannungsverhältnis zur Forderung der AfD nach mehr direkter Demokratie stehen.

\section{Ausgangslage: Die AfD in der politischen Arena}

Im Jahr 2010 wurde »alternativlos « von der Gesellschaft für deutsche Sprache zum Unwort des Jahres gewählt. Vor »Integrationsverweigerer« und »Geschwätz des Augenblicks « war es damit in besonderem Maße von »Sprechern entweder gedankenlos oder mit kritikwürdigen Intentionen verwendet [worden], und dies im öffentlichen Kontext. « ${ }^{38}$ Die meisten dieser sprachstilistischen Fehlleistungen sind innerhalb relativ kurzer Zeit wieder vergessen, im Falle von »alternativlos « ist dies nicht zu erwarten. Hierfür gibt es zwei Gründe:

1. wurde es jeweils in einem expliziten wirtschaftlichen Zusammenhang, von der ersten Frau an der Spitze der Regierung Großbritanniens und Deutschlands verwand,

2. führte es zumindest nach Aussagen der maßgeblichen Akteure zur Gründung einer neuen Partei in Deutschland, welche in ihrem eigenen Namen die Gegnerschaft zu diesem Prinzip ausdrückt.

Beim ersten Punkt entsteht der Eindruck, dass sich Geschichte wiederholt; praktisch bringt die deutsche Bundeskanzlerin Angela Merkel im Zuge der Eurokrise nur eine von Margaret Thatcher erprobte Strategie - das »Tina«-Prinzip (There is no Alternative) $)^{39}$ - zum Einsatz. Die Alternative, die die AfD bieten möchte, sieht sich in Opposition zur Wirtschafts- und mehr noch der Währungspolitik der EU. Dabei sind die Ursprünge der Euro-Kritik in Deutschland allerdings älter. So hat u. a. bereits der Bund freier Bürger (BFB) Mitte der 1990er Jahre, unter Mitwirkung von Joachim Strabatty, vor den »Ge-

38 Zur Liste der Unwörter seit 1991 siehe: Gesellschaft für deutsche Sprache (Hg.), Unwörter des Jahres (<http://www.gfds.de/aktionen/wort-des-jahres/unwoerter-des-jahres/> am 13.7.2014). Die Auswahlkriterien werden von der seit 1994 aktiven Jury bestimmt (<http://w ww.unwortdesjahres.net/> am 16.7.2014).

39 Der Ursprung dieser Argumentation findet sich, nach dem Kenntnisstand der Autoren in folgender Pressekonferenz: Margaret Thatcher Foundation (Hg.), Press Conference for American Correspondents in London (<http://www.margaretthatcher.org/Speeches/displaydocument.a sp?docid=104389\&doctype $=1>$ am 13.7.2014). 
fahren « der damals noch zukünftigen Einheitswährung gewarnt. ${ }^{40}$ So gesehen besteht eine Kontinuität. Tatsächlich unterscheiden sich die gegenwärtigen Rahmenbedingungen für Euro-kritische Parteien immens von jenen in der Mitte der 1990er Jahre. Diese Entwicklungen sind vielgestaltig und komplex und sollen nur in aller Kürze nachgezeichnet werden. Als Ergebnis lässt sich zuvor bereits feststellen, dass diese Veränderung nicht teleologisch zur AfD geführt haben, vielmehr ist sie der Akteur, der es versteht die entstandene Gelegenheit zu nutzen.

Nach dem Ende der Ära Kohl, der Beitritt zum Euro und die Einführung der Währung waren längst beschlossen, führten die umfassenden Reformen der rot-grünen Koalition (Agenda 2010) und die hieraus resultierende "Spaltung " der Sozialdemokratie zu einer Verschiebung auf der linken Seite des politischen Spektrums. Die Nachwehen der Gründung der WASG und die anschließende Fusion mit der SED-Nachfolgepartei PDS zu der Partei Die LINKE führten, aus programmatischen und personellen Gründen, zu einem defekten linken Lager, in dem zwar theoretisch parlamentarische Mehrheiten bestehen, aber praktisch keine regierungsfähige Koalition denkbar ist. Die inzwischen von Angela Merkel geführte CDU passte sich an, nachdem ein eindeutig neoliberales Wahlprogramm 2005 beinahe eine Wahlniederlage bedeutet hatte, und nutzte die Chance, Themen zu besetzen, die nicht Bestandteil ihrer traditionellen Programmatik waren. Die Kanzlerin schuf mit der Politik der »kleinen Schritte «, »dem Fahren auf Sicht« und der asymmetrischen Demobilisierung einen Stil, der ihr Erfolge sicherte sowie letztlich die Union zur politischen Mitte rückte und ihr damit bei der Bundestagswahl 2013 eines der besten Ergebnisse der Parteigeschichte bescherte. ${ }^{41}$

Dabei kann nicht außer Acht gelassen werden, dass es im Erfolg wenig beachtete Opfer gegeben hat. Dazu gehört insbesondere die FDP. Aber auch die Integrationskraft der Union ist durch die Reduzierung auf die Person Angela Merkel im Schwinden begriffen. Hinzu kommt, dass in den letzten Jahren das Regierungs- sowie Parlamentshandeln durch die Eurokrise überzeichnet wurden. Durch die proklamierte »Alternativlosigkeit« und das Abstimmungsverhalten der im Bundestag vertretenen Parteien $^{42}$ sowie die Nicht-Thematisierung durch die Oppositionsparteien, welche potentiell eine neue Re-

40 Vgl. Alexander Häusler, Die "Alternative für Deutschland «: Eine neue rechtspopulistische Partei? Materialien und Deutungen zurvertieften Auseinandersetzung, Düsseldorf 2013, S. 26-31; Uschi Götz, »AfD ringt um Ausrichtung bei Europawahl « in: Deutschlandfunk (<http://ww w.deutschlandfunk.de/kandidatensuche-afd-ringt-um-ausrichtung-bei-europawahl.862.de.ht ml?dram:article_id=274164> am 16.7.2014).

41 Siehe exemplarisch: Frank Decker / Eckhard Jesse (Hg.), Die deutsche Koalitionsdemokratie vor der Bundestagswabl 2013, Baden-Baden 2013; Eckhard Jesse / Roland Sturm (Hg.), Bilanz der Bundestagswabl 2013. Vorraussetzungen, Ergebnisse, Folgen, Baden-Baden 2014; KarlRudolf Korte (Hg.), Die politischen Parteien der Bundesrepublik Deutschland (Schriftenreihe), Baden-Baden 2012ff sowie Teil IV »Parteianalysen« in: Oskar Niedermayer (Hg.), Handbuch Parteienforschung, Wiesbaden 2013.

42 Die Partei Die LINKE stimmte gegen Maßnahmen die nach ihrer Ansichts nicht für Menschen sondern für Banken gemacht würden. Doch anders als die Diskussion auf der linken Seite des Spektrums ist die Verbindung mit nationaler Souveränität und Glaubwürdigkeit das »bessere« Angebot für einen Euro-kritischen Protestwähler. 
gierung nach der Bundestagswahl 2013 hätten stellen können, wurde eine offene Flanke im Parteienwettbewerb geschaffen: eine Gelegenheit für eine Partei rechts der Union. ${ }^{43}$

Im Herbst/Winter des Jahres 2012 formierte sich neuerlich Widerstand gegen die Europolitik. Dabei kam es, nach einem wenig erfolgreichen Intermezzo als Wahlalternative 2013 in Verbund mit den Freien Wählern zur Landtagswahl in Niedersachsen, im Februar 2013 zur Gründung der Alternative für Deutschland. ${ }^{44}$ Von Anfang an genoss die Partei große Aufmerksamkeit von Seiten der Medien. Über den Gründungsparteitag wurde ähnlich ausgiebig berichtet wie über den ersten Parteitag der Piraten nach deren Einzug in das Berliner Abgeordnetenhaus 2011. Umfragen belegen bereits in dieser Frühphase, dass sich die Partei auf eine mehr oder weniger klare Nachfrage bei den Wählern stützen kann. ${ }^{45}$

Schaut man auf die Wahlergebnisse in der Folge dieser Gründung (siehe Tabelle 2), fallen die Ergebnisse bei den drei Wahlen im September ganz unterschiedlich aus. Der Landesverband Bayern war nicht in der Lage, sich an der Wahl im Freistaat am 15. September zu beteiligen - oder die AfD wollte vor dem Hintergrund des besonderen Parteienwettbewerbs in Bayern eine Woche vor der Bundestagswahl ganz bewusst einen Stimmungstest vermeiden. ${ }^{46}$

Ungeachtet des Scheiterns an der Fünf-Prozent-Hürde gelang dann eine Woche später der Paukenschlag: Mit 4,7 Prozent erzielte die AfD das stärkste Ergebnis einer neuen Partei auf Bundesebene seit 1949. Sie gewann dabei besonders viele Stimmen aus dem bürgerlichen Lager und stellte sich insgesamt als Protestvehikel dar. ${ }^{47}$ Das Ergebnis bei der gleichzeitig in Hessen stattfindenden Landtagswahl blieb mit 4,1 Prozent deutlich unter dem Bundesschnitt.

Nach dem Achtungserfolg kam ein langer Winter, in dem die Partei an ihrer Ausdifferenzierung arbeitete und sich medial immer wieder den Vorwurf eines rechtspopulistischen Gestus gefallen lassen musste. ${ }^{48}$ Die programmatische Ausdifferenzierung hin

43 Hierzu u. a.: Roland Sturm, »Doch nicht salternativlos<? Die EU-Krisenpolitik im Parteienwettbewerb « in: Jesse / Sturm (Hg.), Bilanz der Bundestagswabl 2013, aaO. (FN 41), S. 511-530; Andreas Wirsching, Der Preise der Freibeit. Geschichte Europas in unserer Zeit, München 2012, S. 392-409.

44 Vgl. Oskar Niedermayer, »Aufsteiger, Absteiger und ewig sonstige teien bei der Bundestagswahl 2013 " in: Zeitschrift für Parlamentsfragen 45, Nr. 1 (2014), S. 73-93, hier S. 88-92.

45 Vgl. Köcher, »Alternative für Deutschland?«, aaO. (FN 7).

46 Vgl. o. A., Rückzug: Anti-Euro-Partei tritt nicht zur Bayern-Wahl an« in: Spiegel-Online (<http://www.spiegel.de/politik/deutschland/anti-euro-partei-tritt-nicht-zur-bayern-wahl-a n-a-899354.html > am 16.7.2014).

47 Dies lässt sich besonders am Zeitpunkt der Wahlentscheidung und an der politischen Gesinnung der Spätentschlossenen zeigen: Rüdiger Schmitt-Beck, „Euro-Kritik, Wirtschaftspessimismus und Einwanderungsskepsis: Hintergründe des Beinah-Wahlerfolges der Alternative für Deutschland (AfD) bei der Bundestagswahl 2013 « in: Zeitschrift für Parlamentsfragen 45, Nr. 1 (2014), S. 94-112.

48 Hierzu beispielhaft: Justus Bender, »Putschversuche« in: Frankfurter Allgemeine Zeitung, 30.11.2013; ders., »Drohung, Verleumdungen, Unterstellungen « in: Frankfurter Allgemeine Zeitung, 2.1.2014; ders., »Dieser Weg wird kein leichter sein« in: Frankfurter Allgemeine Zeitung, 20.5.2013. 
zur Europawahl wurde mit großem Aufwand betrieben, dabei wuchs das vierseitige Bundestagswahlprogramm auf 25 Seiten für die Europawahl. ${ }^{49}$ Formulierungen und Themenansprache bemühen sich insgesamt redlich um einen seriösen Ton, die Forderungen - beispielsweise nach dem Ausstieg aus dem Euro -werden mit wohl gewählten Worten präsentiert. ${ }^{50}$ Institutionell war der Partei der Einzug in Europaparlament mit dem Wegfall der Sperrklausel sicher.

Das Abschneiden bei den zeitgleich stattfindenden Kommunalwahlen kann in mancher Hinsicht als Beleg für die europapolitischen Schwerpunktsetzung der Partei sowie die noch nicht voll ausgeprägten Strukturen auf der kommunalen Ebene gesehen werden.

Tabelle 2: Wablergebnisse der AfD

\begin{tabular}{|l|c|c|c|}
\hline Wahl & $\begin{array}{c}\text { Wahlkreis } \\
\text { bzw. Erstst. }\end{array}$ & $\begin{array}{c}\text { Listen- } \\
\text { bzw. } \\
\text { Zweitst.* }\end{array}$ & $\begin{array}{c}\text { Listen- bzw. } \\
\text { Zweitst. (in \%) }\end{array}$ \\
\hline LTW Niedersachsen 2013** & 39.132 & 39.714 & 1,1 \\
\hline LTW Bayern 2013 & - & - & - \\
\hline LTW Hessen 2013 & 42.721 & 126.906 & 4,1 \\
\hline Bundestagswahl 2013 & 810.915 & 2.056 .985 & 4,7 \\
\hline KW Baden-Württemberg 2014*** & - & - & - \\
\hline KW Brandenburg 2014 & - & 109.070 & 3,9 \\
\hline KW Hamburg 2014*** & - & - & - \\
\hline KW Mecklenburg-Vorpommern & - & 77.263 & 4,2 \\
\hline 2014 & - & 179.398 & 2,6 \\
\hline KW Nordrhein-Westfalen 2014 & - & 51.555 & 3,0 \\
\hline KW Rheinland-Pfalz 2014 & - & 22.459 & 5,3 \\
\hline KW Saarland 2014 & - & 266.829 & 5,7 \\
\hline KW Sachsen 2014 & - & 55.273 & 2,3 \\
\hline KW Sachsen-Anhalt 2014 & - & - & - \\
\hline KW Thüringen 2014*** & - & 2.070 .014 & 7,1 \\
\hline Europawahl 2014 & 105.024 & 159.611 & 9,7 \\
\hline LTW Sachsen 2014 & & & \\
\hline
\end{tabular}

49 Die Schritte von den ersten Europapolitischen Thesen über diverse und umfängliche Zwischenschritte, inklusive einer Mitgliederbefragung, können hier aus Platzgründen nicht dargestellt werden. Im Rahmen der »Political Linguistics III « Konferenz in Warschau wurde diese Entwicklung durch die Autoren vorgestellt und wird voraussichtlich 2015 im zugehörigen Sammelband veröffentlicht.

50 Vgl. Nestler / Schütt, »Die Europawahl 2014 in Mecklenburg-Vorpommern«, aaO. (FN 1), S. 24 . 


\begin{tabular}{|l|c|c|c|}
\hline Wahl & $\begin{array}{c}\text { Wahlkreis } \\
\text { bzw. Erstst. }\end{array}$ & $\begin{array}{c}\text { Listen- } \\
\text { bzw. } \\
\text { Zweitst.* }\end{array}$ & $\begin{array}{c}\text { Listen- bzw. } \\
\text { Zweitst. (in \%) }\end{array}$ \\
\hline LTW Brandenburg 2014 & 88.330 & 120.077 & 12,2 \\
\hline LTW Thüringen 2014 & 20.833 & 99.545 & 10,6 \\
\hline
\end{tabular}

Quelle: Eigene Zusammenstellung nach den jeweiligen amtlichen Endergebnissen. * Für die Kommunalwahlen ist, durch die Möglichkeit des Kumulieren und Panaschierens, die tatsächliche Anzahl von AfD-Wählern nicht zu ermitteln. ** In Niedersachsen war noch die sogenannte Wahlalternative 2013 zusammen mit dem Freien Wählern angetreten. *** Über die Landeswahlleiter BadenWürttemberg, Hamburg und Thüringen sind nur Zahlen für die etablierten Parteien zu erhalten.

Die AfD hat mit ihren bisherigen Ergebnissen und ihrer politischen Ausrichtung durchaus Chancen sich zu etablieren. Die Landtagswahlen im August und September 2014 in drei ostdeutschen Bundesländern bestätigen den bisherigen Trend: Sowohl bei der Bundestags- als auch bei der Europawahl konnte sie dort besser abschneiden als im Westen der Republik. Die verinnerlichten Deutungsmuster beeinflussen als Filter gleichermaßen die Bewertung von Problemlagen und die Wahrnehmung verfügbarer Handlungsoptionen und -motive. Das wichtigste Element für einen denkbaren Erfolg ist deren öffentliche Kommunikation.

\section{Das diskursive Habitat der AfD}

\subsection{Virulente Diskurse, die die Partei bedient}

Die AfD bedient, in ihren offiziellen Dokumenten wie in öffentlichen Äußerungen ihrer Sprecherfiguren, mehrere momentan in der Gesellschaft virulente Diskurse, um Wähler und Unterstützer mit unterschiedlichen Präferenzen zu erreichen. Sie zehrt davon, dass bestimmte Deutungs- und Wertungsschemata nicht nur präsent, sondern zudem bei jeweils einer bestimmten - mehr oder minder großen - Klientel anerkannt sind. Die Sprecherfiguren der AfD beziehen sich auf diese Diskurse in der nicht unbegründeten Hoffnung, damit auch die jeweilige Klientel für sich einnehmen und mobilisieren zu können. Besonders prominent ist das Anknüpfen an die folgenden Diskurse:

Postdemokratie. Seit den 1990er Jahren ist in der Bundesrepublik von »Politikverdrossenheit« die Rede, die an sinkender Wahlbeteiligung, am Mitgliederschwund der Parteien und am Verlust des Vertrauens in Parlamente und Parteien festgemacht wird. ${ }^{51}$ Seit einigen Jahren genießt zudem die griffige Gegenwartsdiagnose der Postdemokratie in Deutschland große Popularität quer durch die politischen Lager, vor allem im Anschluss an das gleichnamige Buch von Colin Crouch. ${ }^{52}$ Die westlichen Demokra-

51 Für eine kritische, differenzierte Untersuchung der These von der wachsenden Politikverdrossenheit in Deutschland mit aktuellen Zahlen siehe: Thomas Petersen / Dominik Hierlemann / Robert B. Vehrkamp / Christopher Wratil, Gespaltene Demokratie. Politische Partizipation und Demokratiezufriedenheit vor der Bundestagswabl 2013, Gütersloh 2013.

52 Colin Crouch, Postdemokratie, Frankfurt am Main 2008. 
tien verkommen demnach immer mehr zu reinen institutionellen Fassaden, in denen die Wähler zu passiven Zuschauern eines Medienspektakels degradiert werden. Während sie mit Symbolpolitik abgespeist würden, seien die maßgeblichen Entscheidungen hinter verschlossenen Türen von Berufspolitikern und Lobbyisten längst getroffen. Die Klage über eine politische Rhetorik der Alternativlosigkeit gehört ebenso zur PostdemokratieDiagnose wie der Ruf nach Demokratisierung durch mehr Bürgerbeteiligung.

Die AfD schlägt Profit aus dem Revival, das Bürgerbeteiligung und Partizipation derzeit angesichts der verbreiten Wahrnehmung postdemokratischer Zustände erleben. ${ }^{53}$ Nur zu deutlich macht sie sich das populistischen Potenzial der Postdemokratie-Diagnose zu nutze. Sie präsentiert sich als Anti-Parteien-Partei, als die natürliche Verbündete der Bürger gegen das Kartell der »Altparteien«, wie sie in der Diktion der AfD gern genannt werden. Auf der Ebene der EU und im Rahmen der Euro-Rettungspolitik potenziert sich der Vorwurf der Postdemokratisierung freilich noch. Mit der Kompetenzverlagerung von den Nationalstaaten an supranationale Institutionen steht die EU bei der AfD geradezu sinnbildlich für die Entmachtung der Bürger ${ }^{54}$ Allerdings sind die »politische Klasse« und »die Medien « nur ein Teil des Feindbildes. Nicht minder werden organisierte Interessen unter Generalverdacht gestellt, gegen den eigentlichen Willen des Volkes ihren Einfluss geltend zu machen. ${ }^{55}$ Wie jede Form des Populismus beschwört auch die AfD das Fantasma des einfachen, moralisch integren Volkes, das unbekümmert leben könnte, wäre es nicht durch »böswillige Mächte« bedrängt. ${ }^{56}$

Neoliberalismus. Nicht nur im Hinblick auf die populistische Stoßrichtung, sondern auch in ihrer positiven Rezeption des Neoliberalismus unterscheidet sich die AfD von der Postdemokratie-Interpretation von Crouch. Zum Markenkern des Neoliberalismus gehören arbeitgeberfreundliche Politik und Flexibilisierung der Arbeitsverhältnisse, private Vorsorge statt Sozialstaat, De-Regulierung und Privatisierung, mehr dem ökonomischem Wettbewerb überlassen, weniger auf politische Steuerung setzen. In Deutschland wird unter dem Etikett einer Rückkehr zur sozialen Marktwirtschaft verstärkt seit Ende der 1990er öffentlich für politische Reformen in diese Richtung geworben. ${ }^{57}$ Der

53 Vgl. Franz Walter, »Bürgerlichkeit und Protest in der Misstrauensgesellschaft « in: Stine Marg / Lars Geiges / Felix Butzlaff / Franz Walter (Hg.), Die neue Macht der Bürger. Was motiviert die Protestbewegungen, Reinbek bei Hamburg 2013, S. 301-344, hier: S. 328f. sowie die weiteren Beiträge ebenda.

54 Das bürokratische »Monster Brüssel« ist ein bestens eingeführter Topos, nicht in offiziellen Rhetorik, aber in den Sympathisantenkreisen der Partei wird dementsprechend auch gern gegen die »EUdSSR «gewettert.

55 Ein klassischer Topos nicht nur konservativer Parlamentarismuskritik, die fast wortgleich schon bei Carl Schmitt (Die geistesgeschichtliche Lage des heutigen Parlamentarismus, München/Leipzig 1926) zu finden ist. Zur Rolle des Parlaments in Krisendiagnosen siehe auch: Markus Linden, »Die Rolle des Parlaments in Krisendiagnosen des 20. und 21. Jahrhunderts « in: Markus Linden / Winfried Thaa (Hg.), Krise und Reform politischer Repräsentation, BadenBaden 2011, S. 149-172.

56 Hierzu: Lars Rensmann, »Populismus und Ideologie« in: Decker (Hg.), Populismus. Gefabr für die Demokratie oder nützliches Korrektiv, aaO. (FN 25), S. 59-80.

57 Vgl. Rudolf Speth, Die politischen Strategien der Initiative Nene Soziale Marktwirtschaft, Düsseldorf 2004 sowie ders., Die zweite Welle der Wirtschaftskampagnen, Düsseldorf 2006. 
bundesrepublikanische Gründungsmythos wird nicht ohne Erfolg gegen den keynesianisch-sozialdemokratischen Sozialstaat in Stellung gebracht. ${ }^{58}$

Führende Köpfe der AfD wie Bernd Lucke oder Joachim Starbatty sind Wirtschaftsprofessoren mit ausgeprägtem wirtschaftsliberalen Profil, die sich aktiv an der Propagierung marktliberaler Reformen beteiligen. ${ }^{59} \mathrm{Zudem}$ fügt sich die neoliberale Kritik an einem ausufernden, zentralistischen Sozialstaat, der mit einer überbordenden Bürokratie private Initiative unterbindet, bei der AfD nahtlos in die Diagnose von den politisch entmachteten Bürgern ein. Nicht minder wird die ökonomische Inkompetenz und ideologische Verbohrtheit der »politischen Klasse« gegeißelt. Die EU und besonders der Euro werden so etwa als »politisch motivierte Entscheidung jenseits ökonomischer Vernunft $\ll^{60}$ verdammt. Während die »Altparteien « inklusive CDU/CSU und FDP vom Pfad der marktliberalen Tugend abgekommen seien, verbleibe allein die AfD als Vorkämpferin für Marktwirtschaft und Subsidiarität als Korrektiv zum »Zentralismus «.

Allerdings melden sich auch interne Kritiker zu Wort, die das marktliberale Profil der Partei gefährdet sehen, wie etwa die so genannten Kolibris (Konservative und Liberale in der AfD), die sich Anfang 2014 um Starbatty und Dagmar Metzger als Gruppe innerhalb der AfD formiert haben. Überraschend trat Metzger im März dieses Jahres von ihrem Posten als Pressesprecherin der Partei zurück. Im April veröffentlichte eine lose Gruppe der Liberalen in der AfD ein Positionspapier, dass die Betonung der Freiheit im öffentlichen Auftritt der Partei anmahnt, wobei in den inhaltlichen Forderungen Freiheit wiederum vorwiegend neoliberal verstanden wird. ${ }^{61}$ Auf Unverständnis stieß auch Luckes Distanzierung von einer Aussage Hans-Olaf Henkels auf einer Pressekonferenz zur Europawahl. Der frühere BDI-Präsident und Listenzweiter der AfD zur Europawahl hatte seine neue politische Heimat als die »liberalste aller Parteien « und die »letzte liberale Partei Deutschlands« bezeichnet.

Nationalchauvinismus. Genauso wird in der Partei, bisweilen von ein und denselben Sprechern, auf der Klaviatur nationalchauvinistischer Ressentiments gespielt. Die Weitergabe von Kompetenzen an die EU wird dann als externer Angriff auf die Souveränität des deutschen Volkes beschrieben. Zugleich schürt die AfD die Angst vor dem Verlust

58 Trotz der allgemein hohen Zustimmung zur sozialen Marktwirtschaft belegen Umfragen aber auch ein Unbehagen an mangelnder sozialer Gerechtigkeit - gerade angesichts der Wirtschaftskrise, vgl. Institut für Demoskopie Allensbach, Einstellungen zur Sozialen Marktwirtschaft in Deutschland am Jabresanfang 2010, Allensbach 2010; Infratest dimap, ARD-Deutschlandtrend Februar 2012 (<http://www.infratest-dimap.de/ 2012/februar/> am 13.7.2014).

59 Z. B. Starbattys Aktionsgemeinschaft Soziale Marktwirtschaft oder der Aufruf zur Ernenerung der Sozialen Marktwirtschaft (2008) oder auch Luckes Hamburger Appell (2005), der maßgeblich durch eine Anzeigenkampagne der »Initiative Neue Soziale Marktwirtschaft « verbreitet wurde.

60 So z. B. in den Thesen der Partei zur Europawahl.

$61 \mathrm{Zu}$ den Urhebern gehören der erst kürzlich in den Bundesvorstand gewählte Marcus Pretzell und Philipp Ritz, Vorsitzender der Jungen Alternative. Dokumentiert Online (<http://ef-ma gazin.de/2014/04/01/5141-dokumentation-waehrungswettbewerb-und-steuerfreiheit $>$ am 13.7.2014). 
nationaler Identität. ${ }^{62}$ In aller Klarheit erteilt die Partei so einer »europäischen Identität « eine Absage. Das Projekt einer vertieften supranationalen Integration, missachte die tatsächlichen Gegebenheiten und historischen Erfahrungen auf dem Kontinent. ${ }^{63}$

Zudem wird Stimmung gemacht gegen die angeschlagenen Euro-Länder. Die Mitgliedschaft in der Euro-Zone mache das wirtschaftlich starke Deutschland unweigerlich zum Zahlmeister der Staaten, die weitgehend selbstverschuldet in Not geraten seien. Das Bild der faulen Südländer ist nicht nur durch die Arbeit reißerischer Druckerzeugnisse von der Bild-Zeitung bis zu Thilo Sarrazins Bestsellern gut eingeführt, auch die sonst meist um Nüchternheit bemühte Bundeskanzlerin Angela Merkel hat sich schon dieser Stereotype bedient. ${ }^{64}$

Unweigerlich werden in diesem Zusammenhang auch bestehende Ressentiments gegenüber Migranten bedient. Dabei wird einerseits versucht, den Schein der Seriosität zu wahren, wenn die Notwendigkeit von »qualifizierter « Zuwanderung nach dem kanadischen Modell betont wird. Andererseits wird zielsicher im selben Atemzug das Szenario von Einwanderermassen heraufbeschworen, die allein mit der Absicht nach Deutschland strömten, um auf Kosten des hiesigen Sozialstaats zu leben. ${ }^{65}$ Das Inkrafttreten der Arbeitnehmerfreizügigkeit für Bulgaren und Rumänen in der EU Anfang 2014 wurde nicht nur von der AfD zum Anlass genommen, diese Ressentiments zu schüren. ${ }^{66}$

Die AfD bekennt sich in ihren jüngst veröffentlichten »Politischen Leitlinien « zur Westbindung und zur NATO. Zugleich wird diese Position konterkariert durch die Forderung nach einer an nationalen Interessen orientierten Macht- und Realpolitik. Vor allem Alexander Gauland, stellvertretender Vorstand, tut sich auch auf der offiziellen Website in dieser Hinsicht hervor. ${ }^{67}$ Offen redet er über eine Neuauflage Bismarckscher Politik der Balance of Power, was im Endeffekt nichts anderes bedeutet als die Rückkehr zu einem Europa, in dem sich die Länder nicht mehr als Partner verstehen, sondern wieder als Gegner und potentielle Feinde. ${ }^{68}$ Seine andernorts ebenfalls im Anschluss an Bismarck getroffene Feststellung, die großen Fragen der Zeit würden immer noch mit »Eisen

62 Eher skurril ist in dieser Hinsicht die Forderung des AfD-Landesverbandes Sachsen nach einer Deutschquote im Radio. Ungleich ernster zeigen sich die Verlustängste, wenn im gleichen Wahlprogramm der Bau von Minaretten an einen positiven Volksentscheid geknüpft wird. Siehe AfD Sachsen, Wablprogramm 2014.

63 Ein entsprechender Passus steht am Anfang der Thesen zur Europawahl 2014, in der endgültigen Fassung wurde auf diese Formulierung verzichtet.

64 Vgl. Reiner Wandler, »Die Kanzlerin und die `Südländer«. Mit Merkel am Ballermann« in: taz (<http://www.taz.de/!71032/> am 14.7.2014).

$65 \mathrm{Zu}$ Recht wurde darauf hingewiesen, dass die AfD dabei ein Vokabular bemüht, das demjenigen der NPD fast aufs Wort gleicht, wenn etwa der "Einwanderung in die Sozialsysteme " (Parteiproramm 2013, Entwurf Politische Leitlinien 2014) eine Absage erteilt wird oder klargestellt wird, dass Deutschland nicht das »Sozialamt (Wablplakat zur Europawabl 2014) der Welt sei.

66 So beispielsweise die CSU in Person des ehemaligen Innenministers Hans-Peter Friedrich (<http://www.spiegel.de/politik/deutschland/innenminister-friedrich-will-schengen-erweite rung-blockieren-a-886592.html> am 14.7.2014).

67 Ähnlich auch die Patriotische Plattform, die sich Anfang 2014 in der Partei gegründet hat.

68 Alexander Gauland, Thesenpapier zur Außenpolitik, (<https://www.alternativefuer.de/2013/ 09/11/thesenpapier-aussenpolitik> am 14.7.2014).

ZfP 61. Jg. 4/2014 
und Blut « entschieden, ${ }^{69}$ mag für das Gros der Deutschen wenig verlockend klingen. Im Hinblick auf die Ukraine-Krise 2014 kommt Gaulands außenpolitische Position jedoch einer weitverbreiteten isolationistischen Stimmung in Deutschland entgegen. Wenn man die Annexion der Krim durch Russland nicht als völkerrechtswidrigen Akt versteht, sondern als normalen Vorgang in den internationalen Beziehungen ${ }^{70}$ oder gar als zu erwartende Antwort auf eine aggressive EU-Osterweiterung, dann können sich die Deutschen heraushalten, Putins zynische Propaganda geradezu dankbar für bare Münze nehmen und sich obendrein noch als Hüter des Weltfriedens fühlen. ${ }^{71}$ Allerdings ist diese Lesart in der Partei auch nicht unumstritten. ${ }^{72}$

Kritik der Political Correctness. Neben der Euro-Kritik hat sich die Selbststilisierung zum Opfer von »Gutmenschen« und »Tugendwächtern« zum Markenkern der AfD entwickelt. Die Partei reiht sich damit ein in die Riege von selbst ernannten Hütern der Meinungsfreiheit, die den Auftritt zur besten Sendezeit und in den auflagenstarken Zeitungen dazu nutzt, die (Selbst-)Zensur eben jener so genannten Mainstreammedien zu beklagen. Dabei werden Kritik und Widerworte umgedeutet in den Versuch, den Künder unbequemer Wahrheiten mundtot zu machen. Jeder, dessen öffentliche Äußerungen kritisiert werden, egal wie abstrus diese sind, kann sich so als Märtyrer für die Meinungsfreiheit gerieren, die von einem irgendwie linken Autoritarismus bedroht ist. Interessanterweise waren es in den letzten Jahren Konservative, die vor der Bedrohung der bürgerlichen Mitte der Gesellschaft gewarnt und der »veröffentlichten « Meinung des Mainstreams im Namen der tatsächlichen »öffentlichen« Meinung den Kampf angesagt haben. Thilo Sarrazin ist lediglich der bekannteste - und erfolgreichste - Vertreter dieser

69 Alexander Gauland, »Warum sich die Deutschen mit Gewalt so schwer tun«, Der Tagesspiegel 23.7.2012.

70 Alexander Gauland (Rede von Gauland zur Lage in der Ukraine (<http://www.alternativefu er.de/rede-von-gauland-zur-krise-der-ukraine> am 14.7.2014) erläutert den Deutschen in ihrem kosmopolitischen Wolkenkuckucksheim dann auch, das »Einsammeln russischer Erde« sei quasi natürliche Konstante russischer Außenpolitik.

71 Einer von der Körber-Stiftung in Auftrag gegebenen Umfrage (Die Sicht der Deutschen auf die Außenpolitik Eine Studie von TNS Infratest Politikforschung im Auftrag der Körber-Stiftung, Berlin 2014) zufolge waren im April/Mai 201460 Prozent der Deutschen dagegen, dass Deutschland bei internationalen Krisen größere Verantwortung übernimmt. Politbarometer und Deutschlandtrend (Februar bis Juni 2014) legen nahe, dass gut 40 Prozent der Deutschen keine Einmischung Deutschlands in die Ukraine-Krise wünschen. Wie das IfD Allensbach (IfD-Umfrage 11023, April 2014) zudem zeigt, ist diese Stimmung im Osten stärker ausgeprägt als im Westen. Bestätigt fühlen können sich die Deutschen dabei durch den zur moralischen Institution verklärten Altkanzler Helmut Schmidt oder den Putin-Intimus und Altkanzler Gerhard Schröder.

72 Vgl. die Diskussion auf dem Erfurter Parteitag 2014, die Antwort der so Konservativen und Liberalen in der AfD auf Gaulands Ukraine-Rede (<www.kolibri-afd.de/offener-brief-zuraussenpolitik-der-afd/369 sowie www.kolibri-afd.de/putins-neue-breschnew-doktrin/387> am 14.7.2014) und wiederum die Replik darauf von der Patriotischen Plattform (<www.patriotische-plattform.de/erklaerung-der-patriotischen-plattform-zur-afd-aussenpolitik> am 14.7.2014). 
rhetorischen Strategie. ${ }^{73}$ So schlicht diese Rhetorik sein mag, sie scheint durchaus einen Nerv in der Bevölkerung zu treffen. ${ }^{74}$

Anschaulich zeigt die AfD, dass im Grunde jedes Thema in diesem Modus der Selbstviktimisierung bearbeitet werden kann. Wichtiger als die Euro-Krise oder Außenpolitik sind hier noch die Zuwanderung, die Rechte von Homosexuellen und die Geschlechtergerechtigkeit. Besonders bei letzterem Thema kochen die Emotionen regelmäßig hoch. Zum einen stellt sich die AfD gegen jegliche Art von Frauenförderung etwa mittels Quotenregelungen. Zum anderen warnt die Partei davor, dass Tugendwächter es auf eine Umerziehung abgesehen hätten, die die Geschlechteridentitäten ganz aufzulösen trachte. Beides wird - in bemerkenswerter Unkenntnis - unter Gender-Mainstreaming rubriziert, wahlweise auch »Gender-Ideologie« oder gleich »Gender-Wahnsinn«. Die AfD zielt damit auf all jene, die die Kritik am traditionellen Familienbild und an überlieferten Geschlechterrollen als eine existenzielle Bedrohung erfahren. Der Widerstand gegen die urdemokratische Forderung, Diskriminierungen abzuschaffen, wird wiederum präsentiert als Aufstand der aufrechten Demokraten gegen die Phalanx von »politischer Klasse« und »Mainstreammedien«, die im Grunde randständige Interessen anstatt das Gemeinwohl vertreten. ${ }^{75}$

\subsection{Friktionen}

Das diskursive Habitat besteht nicht nur aus den Diskursen, die die Akteure bedienen können, um daraus Profit zu schlagen. Biographische Studien und Analysen der Milieus,

$73 \mathrm{Zu}$ nennen wären hier auch Peter Sloterdijk (»Die Revolution der gebenden Hand «, Eva Hermann, jüngst aber auch Matthias Matussek (»Ich bin homophob und das ist gut so«) oder, Akif Pirincci (»der Irre Kult um Frauen, Homosexuelle und Zuwanderer «); vgl. hierzu auch allgemeiner: Lucke, »Propaganda der Ungleichheit «, aaO. (FN 10), S. 55-63; Volker Weiß, Deutschlands neue Rechte. Angriff der Eliten - von Spengler bis Sarrazin, Paderborn u.a. 2011; Axel Brüggemann, »Im dunkeldeutschen Wald. Die Rechtsdenker« in: Der Freitag, 4.3.2014.

74 Einer von Die Zeit (Lisa Caspari, »Viele AfD-Anhänger wählten früher die Linke und die FDP « in: Die Zeit 17.4.2013) in Auftrag gegebenen repräsentativen Umfrage von YouGov zufolge glaubten im April 201333 Prozent der Deutschen, man dürfe hierzulande nicht zu allen Themen seine Meinung sagen. Unter den 25- bis 34-jährigen waren demnach sogar 43 Prozent dieser Ansicht.

75 Hierzu: Andreas Kemper, Familie als Keimzelle der Nation? Familien- und geschlechterpolitische Positionen der AfD, Berlin 2014. Das (vermutete) Lebensgefühl der Zielgruppe der AfD ist einfühlsam beschrieben in Gaulands Brief an konservative Parteifreunde, (<https://www. alternativefuer.de/2013/12/29/brief-an-konservative-parteifreunde $>$ am 14.7.2014). Frauke Petry waren neben Thilo Sarrazin und Eva Hermann als Headliner bei der von Juergen Elsässer ausgerichteten Compact-Konferenz Für die Zukunft der Familie. Werden Europas Völker abgeschafft? Familienfeindlichkeit, Geburtenabsturz und sexuelle Umerziehung eingeplant. Beatrix von Storch, für die AfD im Europaparlament, betreibt ein weitverzweigtes Netzwerk von Initiativen, Lobby-Organisationen, Websites und Online-Zeitungen, das sowohl für marktradikale wie gesellschaftspolitische ultrakonservative Positionen wirbt. Von Storchs Zivile Koalition e. V., die auch federführend bei der Gründung der AfD und ihren Vorgängergruppierungen war, ist etwa die Ausrichterin einer Initiative Familienschutz, die u. a. an den Protesten gegen den Bildungsplan 2015 in Baden-Württemberg beteiligt ist.

ZfP 61. Jg. 4/2014 
aus denen die führenden Köpfe der AfD stammen, können die ideellen Wurzeln der Partei erhellen. Im gegebenen Rahmen ist diese Aufgabe nicht realisierbar. ${ }^{76}$ Wohl aber können zumindest Aussagen und Aktivitäten der Akteure herangezogen werden, die vor Parteigründung oder Parteibeitritt zu datieren sind. ${ }^{77}$ Hier sollen lediglich jene Wurzeln der Partei interessieren, die mit der offiziellen Parteirhetorik in einem Spannungsverhältnis stehen.

Ökonomische Expertise. In der Führungsriege ist die im Grunde apolitische Überzeugung verbreitet, das politische System und die »politische Klasse« seien zu sehr in ideologische Spiegelfechtereien und parteipolitische Kalküle verwickelt, um die tatsächlichen politischen Probleme noch angehen zu können. Demnach sei die Stunde jener Experten von außerhalb des politischen Systems gekommen, die frei von ideologischem Ballast, in voller Kenntnis der Sachlage vernünftige Lösungen liefern. ${ }^{78}$ Augenscheinlich fühlen sich besonders Ökonomen zu dieser Aufgabe berufen. Man denke etwa an die Reihe von Verfassungsklagen, die Starbatty seit Anfang der 1990er gegen die Maastricht-Verträge, die Einführung des Euros oder den ESM angestrebt hat. Auch das Engagement für AntiParteien-Formationen wie dem BFB, den Freien Wählern, der Wahlalternative 2013 und schließlich der AfD sind Ausdruck dieses Selbstverständnisses.

Dabei spielt es keine Rolle, dass sich diese Expertise in der Praxis der Partei bislang nicht erkennbar niedergeschlagen hat - abgesehen davon, dass entsprechende akademische Titel und Erfahrungen in der »freien Wirtschaft « wie eine Monstranz vor sich hergetragen werden. ${ }^{79}$ Wichtiger ist, dass dieses szientifisch-technokratische Politikverständnis verbunden wird mit dem populistischen Argument, »dichter an den Bürgern« zu sein als die »politische Klasse« und den authentischen »Bürgerwillen« zum Ausdruck zu bringen. Man kann das Verhältnis zwischen »Bürgerwille« und Expertise auf zweierlei Art begreifen und es führt gleichermaßen in argumentative Sackgassen. Entweder geht man davon aus, dass beide auf wundersame Weise dauerhaft konvergieren. Das würde aber die Expertise zu einer bloßen wissenschaftlichen Verbrämung von Alltagswissen machen. Was noch gravierender wäre: die vehement vertretene direkte Demokratie wäre dann nichts weiter als die plebiszitäre Legitimation von Entscheidungen, die gar nicht anders getroffen werden dürfen - und so im Grunde eine Farce.

76 Eine entsprechende Untersuchung ist in Vorbereitung, deren Ergebnisse die Autoren in einer künftigen Publikation vorstellen werden.

77 Hierzu auch: David Bebnowski / Nils Kumlar, »Jeder hat Angst, seinen Besitzstatus zu verlieren. Die Anti-Euro-Proteste« in: Marg / Geiges / Butzlaff / Walter (Hg.), Die newe Macht der Bürger. Was motiviert die Protestbewegungen, aaO. (FN 53), S. 219-249; Häusler, Die "Alternative für Deutschland«: Eine neue rechtspopulistische Partei?, aaO. (FN 40); Andreas Kemper, Rechte Euro-Rebellion: Alternative für Deutschland und Zivile Koalition e. V., Münster 2013; Holger Oppenhäuser, »Demokratische Querfronten? Der neue Rechtspopulismus und die Ambivalenz der direkten Demokratie« in: Prokla 43, Nr. 2 (2013), S 277-295.

$78 \mathrm{Zu}$ dieser vermeintlichen Ideologielosigkeit siehe auch David Bebnowski, »Die Ideologie der Anti-Ideologen « in: Cicero (<http://www.cicero.de/blog/goettinger-demokratie-forschung/ 2013-04-15/alternative-afd-die-ideologie-der-anti-ideologen> am 14.7.2014).

79 So gehört die Währungspolitik auch nicht zu Luckes ausgewiesenen Forschungsfeldern, vgl. Philip Faigle, »Der falsche Ökonom« in: Die Zeit, 23.5.2014. 
Um keinen Deut schlüssiger wird das Bild, stellt man dagegen die Möglichkeit der Kollision von ökonomischer Expertise und Bürgerwillen in Rechnung. Im Konfliktfall müsste einem von beiden der Vorrang eingeräumt werden. Erhält die ökonomische Expertise den Zuschlag, dann wird Politik unweigerlich zu eben jener technokratischen Governance, die die AfD gegenwärtig (nicht ganz zu Unrecht) ganz im Sinne des Postdemokratie-Diskurses kritisiert. ${ }^{80}$ Ist hingegen im Zweifelsfall der »Bürgerwille « ausschlaggebend, gilt demnach all das als bindend, was die Mehrheit will, dann lässt sich der Anspruch kaum aufrechterhalten, eine Politik nicht nur des »Bürgerwillens «, sondern auch der »ökonomischen Vernunft « $z u$ betreiben.

Elitenberrschaft. Nicht nur das ungebrochene Vertrauen, Experten könnten die wesentlichen Fragen entscheiden, offenbart ein letztlich elitäres Politikverständnis. Wiederum zeigt sich ein krasser Gegensatz zur Postdemokratie-Diagnose von Crouch. Dieser setzt auf eine Verbesserung der Responsivität des parlamentarischen Systems, die durch eine stärkere zivilgesellschaftliche Verankerung der Parteien erreicht werden könne. Zumindest einige der führenden Köpfe der AfD folgen einem ganz anderen Leitbild: dem der unabhängigen, integren Männer - gerne aus der »freien Wirtschaft « -, die über den Interessenkonflikten stehend die Geschicke des Staates zum Wohl der größten Zahl lenken. Ein im Grunde apolitisches Ideal aus dem vorletzten Jahrhundert, das nicht nur wenig plausibel ist, ${ }^{81}$ sondern auch mit der Forderung nach mehr Demokratie kaum zu vereinbaren ist.

Zu diesem Ideal passt, dass auch der Topos der bedrohlichen Masse wieder aufgewärmt wird - nunmehr im Gewand des Hartz-IV-Empfängers (am Ende noch mit Migrationshintergrund!). Ohne Umschweife fragt etwa Rainer Vaubel, Mitglied des wissenschaftlichen Beirats, wie die »Leistungseliten « in der Demokratie vor der Masse geschützt werden könnten. ${ }^{82}$ Etwa dadurch, dass das Stimmgewicht der Wähler von ihrem Steuersatz abhängig gemacht werde, antwortet er sich selbst. Oder indem allein die Wohlhabenden das Passivwahlrecht innehaben sollten bzw. durch eine zweite Kammer, die den Reichtum repräsentiert und einer exzessiven Steuerpolitik zu Lasten der »Leistungseliten « in Zukunft ein Riegel vorschiebe. Der Publizist Konrad Adam, einer der drei Parteivorsitzenden hat sich für die Idee ausgesprochen, gleich allen »Nettostaatsprofi-

80 Vgl. hierzu auch die Kritik von Governance-Konzepten aus demokratietheoretischer Sicht bei Claus Offe, »Governance - Empty signifier oder sozialwissenschaftliches Forschungsprogramm? « in: Gunnar Folke Schuppert und Michael Zürn (Hg.), Governance in einer sich wandelnden Welt, Wiesbaden 2008, S. 61-76; Michael Haus, »Governance-Rhetorik und Institutionenpolitik. Politisierung und Depolitisierung in der Konstruktion neuer Praktiken des Regierens « in: Schuppert / Zürn, aaO., S. 95-117; Michael Th. Greven, »Politik als Problemlösung und als vernachlässigte Problemursache. Anmerkungen zum Verhältnis zwischen der policyForschung und einem veränderten Reformverständnis in Gesellschaft und Politikwissenschaft « in: Klaus Dieter Wolf (Hg.) Staat und Gesellschaft - fähig zur Reform?, Baden-Baden 2007, S. 329-340;

81 Was sollte gerade die Lenker von gewinnorientierten Unternehmen dazu bewegen, auf einmal diese Interessen bei Seite zu lassen, wenn sie die politische Arena betreten?

82 Rainer Vaubel, »Schutz der Leistungseliten in der Demokratie« in: wirtschaftlichefreibeit.de (<http://wirtschaftlichefreiheit.de/wordpress/?p=17> am 14.7.2014).

ZfP 61. Jg. 4/2014 
teuren « das Wahlrecht zu entziehen. ${ }^{83}$ Dazu zählen, notabene, nicht nur die ungeliebten Empfänger von Sozialleistungen, sondern auch Rentner, Beamte und Berufspolitiker. ${ }^{84}$

Die AfD hatte sich die Schwächung der Parteien bereits in ihrem ersten Wahlprogramm auf die Fahnen geschrieben. ${ }^{85}$ Ganz in diesem Sinne fordert auch Hans-Olaf Henkel eine Verfassungsreform, die die Direktwahl des Staatsoberhaupts, des Bundeskanzlers und der Ministerpräsidenten ${ }^{86}$ ermöglicht und so von Parteien unabhängigem Personal den Weg in die Ämter ebnet. ${ }^{87}$ Mit ähnlichem Ziel propagiert Jörn Kruse, Wirtschaftsprofessor und Mitglied des wissenschaftlichen Beirats der AfD, seit Jahren die Einrichtung eines Bürgersenats. ${ }^{88}$ Diese dem bisherigen Gesetzgebungsprozess nachgeschaltete Kammer soll aus direkt gewählten, parteiunabhängigen und fachlich kompetenten Abgeordneten bestehen. Erst mit der Zustimmung dieses Gremiums sollen Gesetze Gültigkeit erhalten. Nur konsequent zu Ende gedacht ist dann Hermann Behrends »Mandative Demokratie ${ }^{89}$ Wenn es nach dem Vorsitzenden des NRW-Landesverbandes ginge, bräuchte es gar keine Parteien und Parlamente mehr. Die Regierung könnte direkt gewählt werden, könnte unmittelbar Gesetze erlassen und müsste sich nach Ablauf der Legislaturperiode zur Wahl stellen.

Fraglich ist allerdings, wieweit diese Elitenherrschaftsfantasien von der Parteibasis mitgetragen werden. Immerhin erntet Lucke bereits jetzt parteiintern auch Kritik, einen autoritären Führungsstil zu pflegen. Nicht zuletzt hat ihm die Parteibasis auf dem Parteitag in Erfurt im März 2014 bei dem Versuch, sich als alleiniger Sprecher der AfD zu etablieren, einen Strich durch die Rechnung gemacht. ${ }^{90}$

83 Konrad Adam, »Wer soll wählen? « in: Die Welt, 16.10.2006.

84 Letztlich also auch Professoren und jene Abgeordneten des EU-Parlaments und der Landesparlamente, die die AfD entsendet.

85 Vgl. zu diesem Topos im Milieu der AfD-Unterstützer: Oppenhäuser, »Demokratische Querfronten? Der neue Rechtspopulismus und die Ambivalenz der direkten Demokratie«, aaO. (FN 77).

86 Henkel mag, wie er betont, viel in der Welt herumgekommen sein, die USA werden dadurch jedoch nicht zu einem Beispiel für ein politisches System, in dem das Staatsoberhaupt aus einer Direktwahl ohne Einfluss der Parteien hervorgeht.

87 Hans-Olaf Henkel, »Ein Plädoyer für den mündigen Bürger: Ja zu einer Direktwahl des Bundespräsidenten! in: Konvent für Deutschland, Standpunkte Mai 2009; ders., »Mit neuer Verfassung das Kartell aufbrechen « in: FreieWelt.net (<http://www.freiewelt.net/mit-neuer-verf assung-das-kartell-aufbrechen-10008375/> am 14.7.2014).

88 Ausführlich in: Jörn Kruse, »Das Monopol für demokratische Legitimation und seine Überwindung. Zur konstitutionellen Reform der staatlichen Strukturen« in: Stefan Bayer / Klaus W.Zimmermann (Hg.), Die Ordnung von Reformen und die Reform von Ordnungen: Facetten politischer Ökonomie, Marburg 2008, S. 203-279.

89 Hermann Behrendt, Mandative Demokratie, Düsseldorf 2011.

90 Hierzu u. a.: Melanie Amann, »Chaos-Parteitag in Erfurt. AfD-Chef Lucke spürt den Zorn der Basis « in: Spiegel-Online (<http://www.spiegel.de/politik/deutschland/afd-parteitag-in-e rfurt-lucke-scheitert-an-der-basis-a-960167.html> am 14.7.2014); David Bebnowski, »Souveräner Kopf und subsidiäre Füße« in: Göttinger Institut für Demokratieforschung: Blog (<http://www.demokratie-goettingen.de/blog/souveraner-kopf-und-subsidiare-fuse > am 14.7.2014). 


\section{Fazit}

Eine Angst geht um in Europa und überraschenderweise auch in Deutschland. Der Rechtspopulismus hat mehr oder weniger Konjunktur in den Medien, im Wahlkampf zur Europawahl und an den Wahlurnen am 25. Mai. ${ }^{91}$ Als Indikator dieser Entwicklung ist die AfD als neuer Akteur im bundesdeutschen Parteiensystem auszumachen. Sie changiert zwischen marktliberalen, auf ökonomische Expertise rekurrierenden Positionen und einer nationalkonservativen Rhetorik, die zielsicher tiefsitzende Stereotype und Ressentiments bedient.

Die bisherigen Ausführungen haben gezeigt, dass der einleitend konstatierte »antipopulistische Konsens « im Schwinden begriffen ist. Hierfür sind die folgenden fünf Indikatoren im Beitrag herausgestellt worden: ${ }^{92}$

- In ihrer »alternativlosen« Beantwortung der Krise am Rande der vertraglich fixierten Legitimität konnte die Regierung unter Angela Merkel die Verunsicherungen in der Bevölkerung nicht ausräumen. Eine Entwicklung, die gerade der Komplexität der Rettungsmechanismen, dem Fehlen einer europäischen Öffentlichkeit und dem Konflikt in Politik und Medien zwischen nationalen und supranationalen »Erfolgen « in der EU geschuldet ist.

- Unter AfD-Anhängern wird die Rechtmäßigkeit der Rettungspolitik - Stichwort: »No-Bail Out«-Klausel der EU-Verträge - massiv angezweifelt. Hieraus speist sich die Erosion des Systemvertrauens dieser Gruppe, aber über die mediale Aufmerksamkeit, die dem Thema geschenkt wird, gibt es einen abgeschwächten analogen Trend in der gesamten Bevölkerung. ${ }^{93}$ Aus diesem wahrgenommenen Zerfall des ökonomischen Gefüges und seiner rechtsstaatlichen Garantie nähren sich Abstiegsängste in Teilen der Bevölkerung. Dieser Befund ist besonders ausgeprägt unter AfD-Wählern. Dabei steht Deutschland bislang als Insel der Ruhe in der Krise, wenn nicht als Gewinner da - was in den südeuropäischen Staaten das Bild der »Zuchtmeisterin« Merkel

91 Vgl. Nestler / Schütt, »Die Europawahl 2014 in Mecklenburg-Vorpommern«, aaO. (FN 1) S. 30-32.

$92 \mathrm{Zu}$ quantifizieren ist diese Entwicklung gegenwärtig nur bedingt.

93 Wichtig ist bei dieser Darstellung, dass der Level des Vertrauens im Durchschnitt aller Länder in das Parlament weiterhin höher ist, als in die nationalen Parlamente. Vgl. Europäische Kommission (Hg.), Die öffentliche Meinung in der Europäischen Union, S. 48f. u. 76. Gleichwohl leistet die Zuschreibung von Werten zur Europäischen Union einen Beitrag zur Ausprägung von EU-Vertrauen. »Damit die Union aber als Orientierungs- und Sinnstifter wahrgenommen werden und wirken kann, muss das politische Handel der EU-Eliten die angesprochenen Ideal glaubhaft verkörpern. « Hierzu: Tuuli-Marja Kleiner / Nicola Bücker, Kulturelle Grundlagen der EU-Unterstützung. Der Einfluss von Wertzuschreibungen auf supranationales politisches Vertrauen« in: Politische Vierteljabresschrift 55, Nr. 2 (2014), S. 295-319, hier: S. 315. 
provoziert. Hieraus konstituiert sich ein Bild des »Untergangs des Abendlandes «, der als normal und »richtig « begriffenen Lebenswelt mit ihren Ge- und Verboten. ${ }^{94}$

- Die offene Flanke der etablierten Parteien in EU- und Euro-kritischen Fragen hat die Wahl einer sich hier profilierenden Partei opportun gemacht. ${ }^{95}$ Dabei ist die parteiinterne Darstellung, »dass nun der Durchbruch « gelungen sei und man praktisch »eine neue Volkspartei «96 , fragwürdig. Im Vergleich zur Bundestagswahl profitiert die AfD von der geringeren Wahlbeteiligung und gewinnt lediglich rund 15.000 Stimmen hinzu (siehe Tabelle 2).

- Die Ergebnisse bei den ostdeutschen Landtagswahlen 2014 zeigen, wie erfolgreich die Kommunikationsstrategie der AfD ist. Daneben eröffnen der Abstieg der FDP, die Aufnahme in die Fraktion der Europäischen Konservativen und Reformisten (EKR) und die kommunale Anbahnung einer Koalition mit der CDU hinaus die Chance einer langfristigen Etablierung.

- Schließlich hat sich seit den 1990er Jahren in der Bundesrepublik der Bereich des Denk- und Sagbaren derart verschoben, dass populistische Argumentationen im gesamtgesellschaftlichen Diskurs anschlussfähig geworden sind. Im Besonderen konnte dies gezeigt werden im Zusammenhang der PC-Kritik, des Nationalchauvinismus und des Neoliberalismus. Aber auch die Rede von der Postdemokratie hat zu einer Gewöhnung an die Fundamentalkritik an der "politische Klasse " geführt. ${ }^{97}$

Bei einem intakten anti-populistischen Konsens wäre davon auszugehen, dass entsprechende Sprechakte nicht in den Hauptkanälen des gesamtgesellschaftlichen Diskurses reflektiert - und goutiert - werden, sondern lediglich ein Dasein in Spezialdiskursen fristen.

In der weiterführenden Auseinandersetzung mit dieser Aussage wie mit dem Phänomen der AfD im Allgemeinen bieten sich zwei Wege an, von denen keiner exklusiv beschritten werden muss. Zum einen kann die hier lediglich skizzierte Untersuchung des

94 Vgl. Schmitt-Beck, »Euro-Kritik, Wirtschaftspessimismus und Einwanderungsskepsis: Hintergründe des Beinah-Wahlerfolges der Alternative für Deutschland (AfD) bei der Bundestagswahl 2013 «, aaO. (FN 47); Oliver Decker / Johannes Kiess / Elmar Brähler, Die stabilisierte Mitte. Rechtsextreme Einstellungen in Deutschland 2014, Leipzig 2014, S. 41f. u. 60; Martin Belzer, AfD. Mitgliederbefragung zur Europawabl 2014 (<http://www.alternativefuer.de/bu ndesweite-mitgliederbefragung-zur-europawahl-auswertungen/> am 18.7.2014).

95 In der Bilanz der Bundestagswahl war diese Entwicklung bereits vermutet worden. Hierzu: Roland Sturm, »Doch nicht salternativlos<? Die EU-Krisenpolitik im Parteienwettbewerb « in: Jesse / Sturm (Hg.), Bilanz der Bundestagswabl 2013. Voraussetzungen, Ergebnisse, Folgen, aaO. (FN 41), S. 511-530.

96 So hatte sich Lucke nach Bekanntgabe der ersten Hochrechnung im ZDF geäußert. Hierzu: Nestler / Schütt, »Die Europawahl 2014 in Mecklenburg-Vorpommern «, aaO. (FN 1), S. 26.

97 Diese Entwicklung verändert den politischen Wettbewerb nur bedingt. So haben zwei aktuelle Studien betont, dass weder die Programme der anderen Parteien an Populismus »erkranken « noch das Rechtsaußenparteien den Parteienwettbewerb grundsätzlich beeinflussen. Hierzu: Cas Mudde, »Fighting the System? Populist Radical Right Parties and Party System Change in: Party Politics 20, Nr. 2 (2014), S. 217-226; Matthijas Roodujin / Sarah L de Lange / Wouter van der Burg, »A Populist Zeitgeist? Programmatic Contagion by Populist Parties in Western Europe« in: Party Politics 20, Nr. 4 (2014), S. 563-575. 
diskursiven Habitats durch eine detaillierte Analyse der Sozialisation ausgewählter Sprecherfiguren erheblich ausgebaut werden. Zum anderen ist eine zweigeteilte Medienanalyse ratsam, die sowohl die klassischen Massenmedien als auch die so genannten Social Media umfasst.

\section{Zusammenfassung}

Durch das Zur-Mitte-Rücken der CDU, die Eurokrise und die in zentralen Punkten eiheitliche Reaktion der im Parlament vertretenen Parteien auf diese Krise, ist in Deutschland eine Lücke im Parteienwettbewerb entstanden. Als Beleg hierfür können das Aufkommen der Alternative für Deutschland (AfD) im Frühjahr 2013 und die seitdem mehr oder weniger großen Erfolge dieser Euro-skeptischen Partei gedeutet werden. Dabei setzt sie gezielt Populismus und Selbstviktimisierung als Mittel zur Wählerüberzeugung ein. Der vorliegende Beitrag untersucht die Gelegenheitsstruktur, welche die Partei vorfindet und legt dabei den Schwerpunkt auf die diskursive Ebene. Denn anders als auf die institutionellen, politischen und kulturellen Kontexte können Akteure hier relativ starken Einfluss nehmen.

\section{Summary}

With the CDU orienting towards the centre, with the Euro-crisis and the uniform reaction of the parties represented in the Bundestag to the latter, a representational gap opened within Germany's party system. The rise of the Euro-sceptic Alternative für Deutschland in the spring of 2013 supports the thesis that this gap provides the opportunity for a successful (right-wing) populist party, hitherto deemed next to impossible in Germany. This paper examines the opportunity structure which the party faces, also taking into account the discoursive level. Compared to the institutional, political and cultural level, actors can exert a relatively strong influence on public discourse.

Christian Nestler / Jan Rohgalf, A German Angst - Succesful parties to the right of the Union? AfD and the opportunity structures for right-wing Parties 